\title{
CORRECTION FACTORS FOR DETERMINING THE MASS TRANSFER COEFFICIENTS
}

\author{
GUŽELA Štefan ${ }^{1 *}$, DZIANIK František ${ }^{1}$ \\ ${ }^{I}$ Slovak University of Technology in Bratislava, Faculty of Mechanical Engineering, Institute of Process \\ Engineering, Námestie slobody 17, 81231 Bratislava, Slovak Republic, e-mail: stefan.guzela@stuba.sk
}

\begin{abstract}
A number of industrial operations are linked to mass transfer. The mass transfer coefficient value is necessary to know when designing the industrial equipment in which mass transfer occurs. There are various mass transfer coefficients, as well as equations for their calculation. However, the value of these coefficients determined according to these equations often has to be corrected for the given conditions. The aim of the article is to state the conversion relations - the correction factors enabling the calculation of the mass transfer coefficients values corresponding to the given conditions.
\end{abstract}

KEYWORDS: mass transfer, Fick's law, Stefan's law, Maxwell's law, mass transfer coefficient, concentration diffusion, film theory, correction factor.

\section{Introduction}

From the point of view of industrial practice, mass transfer between the contacting phases is considered to be the most justified case. In this case, one of the phases is often in motion. Mass transfer is generally realized by a molecular and convective mechanisms of mass transfer. When designing the equipment in which mass transfer occurs, both mechanisms of mass transfer should be taken into account. There are various molecular mechanisms of mass transfer $[1,2]$. Concentration diffusion is one of the mechanisms which is dominant applied to a large group of industrial equipment. In the article, the attention is mainly focused on this type of diffusion, where the driving force of the process is the concentration gradient. The equations presented here are valid provided that the process is steady and without a chemical reaction. Knowledge of these equations is important because they are the basis for the design theory of industrial equipment, including those in which the chemical reaction takes place $[3,4]$.

\section{Basic equations describing the concentration diffusion}

The calculation of equipment in which mass transfer occurs due to the concentration diffusion is substantially based on the equation:

$$
\vec{J}_{i}=c_{i}\left(\vec{u}_{i}-\vec{u}^{*}\right)=-c D_{i m} \nabla x_{i}
$$

which is referred to as 1 . Fick's law $[2,5,6]$. In the case of the binary systems, Eq. (1) is written in the form: $\vec{J}_{A}=-D_{A B} \nabla c_{A}$, if $c=$ constant. When mass transfer is realized only along the $z$-axis, then:

$$
J_{A z}=c_{A}\left(u_{A z}-u_{z}^{*}\right)=-D_{A B} \frac{\mathrm{d} c_{A}}{\mathrm{~d} z}
$$


1. Fick's law is a boundary law because this law is exactly valid only for the bidirectional mass transfer (equimolar counter-diffusion) (Fig. 1a). In addition to this type, unidirectional mass transfer (uni-molecular diffusion) (Fig. 1b) also occurs in industrial practice, which represents a boundary case too. This type of mass transfer describes the equation:

$$
J_{A z}=-D_{A B}\left(\frac{c}{c-c_{A}}\right) \frac{\mathrm{d} c_{A}}{\mathrm{~d} z}
$$

which is referred to as Stefan's law [7]. This equation is valid in the case of the binary system, if $c=$ constant, and for mass transfer in the $z$-axis direction.

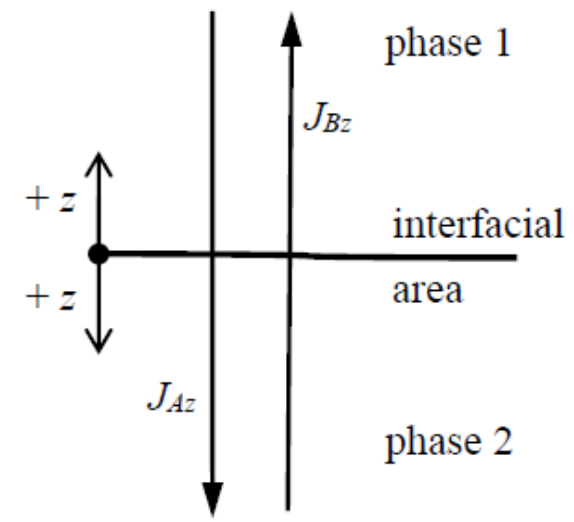

a) Bidirectional mass transfer (equimolar counter-diffusion) (i.e., $J_{A z} \neq 0, J_{B z} \neq 0$, however, $J_{A z}=-J_{B z}$ )

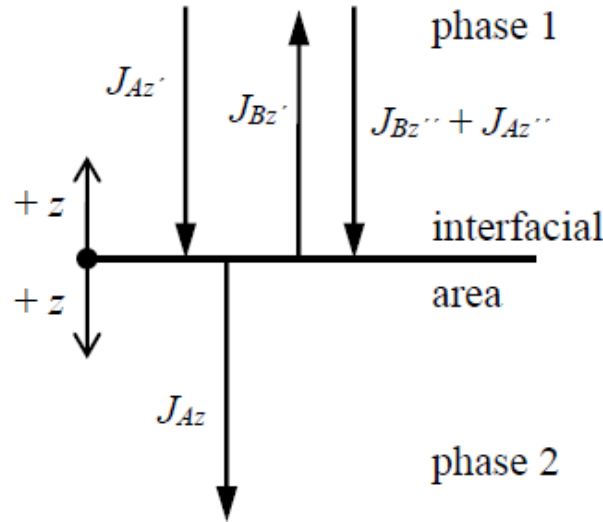

b) Unidirectional mass transfer (uni-molecular diffusion) (i.e., $J_{A z} \neq 0=J_{A z^{\prime}}+J_{A z^{\prime \prime}}, J_{B z}=0$, since, $J_{B z^{\prime}}=-J_{B z^{\prime \prime}}$ )

Fig. 1 Mass transfer (molecular mass transfer) in the binary mixture

These laws, as already mentioned, are boundary. Apparently, there is a general law describing both laws (Fick's and Stefan's law). When the mass transfer is in the $z$-axis direction, then this general law may be written in the form:

$$
-\frac{\mathrm{d} p_{A}}{\mathrm{~d} z}=\beta_{A B} c_{A} c_{B}\left(u_{A z}-u_{B z}\right)
$$

In the literature, this equation represents Maxwell's law [7].

Maxwell's law Eq. (4) enables to describe any molecular mass transfer when the driving force of the process is a concentration gradient. Despite the above, 1. Fick's law often takes precedence in describing the problems based on mass transfer. The advantage of this law is that its form (i.e., Eq. (1), or Eq. (2)) fits very well among the constitutive equations representing the molecular transfer of heat and momentum $[5,6]$. The values obtained in terms of Eq. (3) are often not very different from the results determined in terms of Eq. (2). This special case arises when $c /\left(c-c_{A}\right)=c / c_{B} \cong 1$ in the Eq. (3). In technical practice, this represents a case when the solutions are very diluted (i.e., $c_{A} \rightarrow 0$ ). The ratio $c / c_{B}$ is referred to in the literature as Stefan's correction factor [7].

\section{Molecular mass transfer}

The molecular mass transfer occurs when $u_{z}^{*}=0$. This means that the molar flux of a substance $i$ can be expressed in terms of Eq. (2) as follows: 


$$
J_{i z}=c_{i}\left(u_{i z}-u_{z}^{*}\right)=c_{i} u_{i z}
$$

According to this equation, it is possible to write the expression for $u_{A z}$. Substituting this expression in Eq. (4) for $u_{A z}$, the equation $J_{A z}=c_{A}\left(u_{B z}-\frac{\mathrm{d} p_{A}}{\beta_{A B} c_{A} c_{B} \mathrm{~d} z}\right)$ is obtained, in which $\beta_{A B}=\frac{\mathrm{R}^{2} T^{2}}{p D_{A B}}$ [7]. This equation can be further converted using the Eq into the form:

$$
J_{A z}=x_{A}\left(J_{A z}+J_{B z}\right)-D_{A B} \frac{\mathrm{d} p_{A}}{\mathrm{R} T \mathrm{~d} z}
$$

or when $c=\frac{p}{\mathrm{R} T}=$ constant then the equation $J_{A z}=x_{A}\left(J_{A z}+J_{B z}\right)-D_{A B} \frac{\mathrm{d} c_{A}}{\mathrm{~d} z}$ is obtained. This equation is a special case of the general equation:

$$
J_{A z}=x_{A} \sum_{i=A}^{n} J_{i z}-c D_{A m} \frac{\mathrm{d} x_{A}}{\mathrm{~d} z},
$$

which is valid for $n$ component system. Eq. (7) is the reference equation for describing any problem associated with mass transfer in a stationary environment. It is advantageous to write this equation in the form: $J_{A z}=\left(\frac{-c \gamma^{\prime} D_{A m}}{\gamma^{\prime}-x_{A}}\right) \frac{\mathrm{d} x_{A}}{\mathrm{~d} z}$ where $\gamma^{\prime}=J_{A z} / \sum_{i=A}^{n} J_{i z}$. If $c=$ constant and $D_{A m}=$ constant, the solution to this equation is the expression:

$$
J_{A z}=\frac{-D_{A m}}{\left(z_{2}-z_{1}\right) \varphi^{\prime}}\left(c_{A 2}-c_{A 1}\right)
$$

in which $\varphi^{\prime}=\frac{1}{c \gamma^{\prime}} \frac{c_{A 1}-c_{A 2}}{\ln \left(\frac{c \gamma^{\prime}-c_{A 2}}{c \gamma^{\prime}-c_{A 1}}\right)}$. This equation can be applied to specific cases when the ratio

of the molar fluxes through the interfacial area is known. For example, in the cases of the binary system, if mass transfer is bidirectional (Fig. 1a), it is valid: $J_{A z}=-J_{B z}$ and therefore: $1 / \gamma^{\prime}=0$ and $\varphi^{\prime}=1$; if mass transfer is unidirectional (Fig. 1 b), it is valid: $J_{B z}=0$ and therefore: $1 / \gamma^{\prime}=1$ and $\varphi^{\prime}=\frac{1}{c} \frac{c_{A 1}-c_{A 2}}{\ln \left(\frac{c-c_{A 2}}{c-c_{A 1}}\right)}$.

It should also be noted that the equation in the form:

$$
N_{A z}=x_{A} \sum_{i=A}^{n} N_{i z}-c D_{A m} \frac{\mathrm{d} x_{A}}{\mathrm{~d} z}=c_{A} u_{A z}
$$

is equivalent to the Eq. (7). From this equation, it follows: $N_{i z}=c_{i} u_{i z}$ (or $\vec{N}_{i}=c_{i} \vec{u}_{i}$ ). By comparing this equation with Eq. (5), it is found that $N_{i z}=J_{i z}$ in the case of the molecular mass transfer. By applying just that, Eq. (9) is obtained from Eq. (7). 
Both equations, i.e., Eqs. (7) and (9), represent the constitutive equation of molecular mass transfer by concentration diffusion. In the literature, for example [1, 2], the use of Eq. (9) is generally preferred. In the case of the molecular mass transfer, the quantity $J_{A z}$ in Eq. (7) (or the quantity $N_{A z}$ in Eq. (9)) represents the total molar flux of a substance $A$ through the interfacial area, as well as in the phase itself. This total molar flux is generally the sum of the listed expressions, where expression

$-c D_{A m} \frac{\mathrm{d} x_{A}}{\mathrm{~d} z}=J_{A z^{\prime}}$ represents mass transfer of a substance $A$ as a result of the concentration gradient existence (i.e., the existence of a molecular flux of a substance $A$ ) and

$x_{A} \sum_{i=A}^{n} J_{i z}=J_{A z^{\prime \prime}}\left(\right.$ or $\left.x_{A} \sum_{i=A}^{n} N_{i z}=N_{A z^{\prime \prime}}\right)$ represents mass transfer of a substance $A$ as a result of existence of the molecular flux of the indifferent substances from the interfacial area, which causes so-called a convective flux of a certain amount of substances (mixture) towards the interfacial area, including also a certain proportion of a substance $A$ (i.e., the existence of a convective flux of a substance $A$ ). The fluxes of these indifferent substances are considered to be the same. The convective flux brings such a quantity of the indifferent substances as is just taken away by the molecular flux (see, for example, in Fig. 1b, $J_{B z^{\prime}}=-J_{B z^{\prime \prime}}$ ). As a result of the above, the indifferent substances appear to be relatively immobile.

It follows that, even in the case of the molecular mass transfer, the total molecular mass transfer of a substance $i$ is always generally represented as the sum of the own molecular and convective flux of a substance $i$ (i.e., $J_{i z}=J_{i z^{\prime}}+J_{i z^{\prime \prime}}$ ). It should be remembered that the convective flux of a substance $i$ also applies in cases where $u_{z}^{*}=0$ (i.e., in the molecular mass transfer).

\section{Convective mass transfer}

Convective mass transfer is conditioned by the movement of the fluid as a whole, i.e., the volume flux when $u_{z}^{*} \neq 0$. The description of this type of mass transfer is based on similar equations as in molecular mass transfer. The difference is that, in the above equations, $D_{A m}$ is replaced by $E_{t A m}$ representing the total diffusivity coefficient [1]. By taking this into account, it is possible to write, based on Eq. (9), the general equation for convective mass transfer:

$$
N_{A z}=x_{A} \sum_{i=A}^{n} N_{i z}-c E_{t A m} \frac{\mathrm{d} x_{A}}{\mathrm{~d} z},
$$

in which: $E_{t A m}=D_{A m}+D_{T A m}(z)$. If the fluid flows laminarly, $E_{t A m} \cong D_{A m}$, because: $D_{T A m}(z) \cong 0$. In such cases, the molecular mass transfer is essentially applied. It means, the above equations are used to describe the mass transfer when the fluid flows laminarly. In these equations, then: $N_{A z}=J_{A z}$ (or $N_{i z}=J_{i z}$ ). Conversely, if the fluid flows turbulently, $E_{t A m} \cong D_{T A m}(z)$, then the contribution of turbulent diffusion is dominant. The variable $D_{\text {TAm }}(z)$ represents turbulent diffusivity (eddy diffusivity), which is a function of the diffusion path (i.e., $D_{T A m}=\mathrm{f}(z)$ ). Thus, the total diffusivity $\left(E_{t A m}\right)$ is the sum of the molecular diffusivity $\left(D_{A m}\right)$ and the eddy diffusivity $\left(D_{T A m}(z)\right)$. 


$$
N_{A z}=\frac{-E_{t A m}}{\left(z_{2}-z_{1}\right) \varphi}\left(c_{A 2}-c_{A 1}\right)
$$

in which: $\varphi=\frac{1}{c \gamma} \frac{c_{A 1}-c_{A 2}}{\ln \left(\frac{c \gamma-c_{A 2}}{c \gamma-c_{A 1}}\right)}, \quad \gamma=\frac{N_{A z}}{\sum_{i=A}^{n} N_{i z}}$, and $E_{t A m}=D_{A m}+\bar{D}_{T A m}$. This equation, which is valid under the above assumptions, can be applied to any case of the steady mass transfer, including bidirectional and unidirectional mass transfer. In the case of binary mixtures, it is easy to verify that if $N_{A z}=-N_{B z}$ (bidirectional mass transfer), then $1 / \gamma=0$ and $\varphi=1$. If $N_{B z}=0$ (unidirectional mass transfer), then $1 / \gamma=1$ and $\varphi=\frac{1}{c} \frac{c_{A 1}-c_{A 2}}{\ln \left(\frac{c-c_{A 2}}{c-c_{A 1}}\right)}$. Similar equations apply in the case of molecular mass transfer. By comparing these equations, it is easy to convince that the values of $\gamma$ and $\gamma^{\prime}$, as well as $\varphi$ and $\varphi^{\prime}$, are the same for the same input parameters (i.e., $\gamma^{\prime}=\gamma$ and $\varphi^{\prime}=\varphi$ ). This represents an important fact that Eq. (10) is really a general equation that can also describe the molecular mass transfer when it is valid $E_{t A m}=D_{A m}$ and $N_{A z}=J_{A z}$. Therefore, all further considerations will follow from Eq. (10).

\section{$5 \quad$ Rate equation of mass transfer and mass transfer coefficients}

Eq. (10) can not be solved in general because there is no analytical expression of dependence $D_{T A m}=\mathrm{f}(z)$ [1]. In such cases, practice proceeds by defining a new quantity, which is determined experimentally $[2,5,6]$. This is denoted by the symbol $k_{c}$ and given by the equation:

$$
N_{A z}=k_{c}\left(c_{A 1}-c_{A 2}\right)=-k_{c}\left(c_{A 2}-c_{A 1}\right),
$$

which is said to be rate equation of mass transfer. There are various forms of this equation, because the driving force of the process can be expressed using different concentrations $[2,5,8]$. If this equation is compared with the Eq. (11), the following equation can be written:

$$
k_{c}=\frac{E_{t A m}}{\left(z_{2}-z_{1}\right) \varphi}=\frac{E_{t A m} c \gamma \ln \left(\frac{c \gamma-c_{A 2}}{c \gamma-c_{A 1}}\right)}{\left(z_{2}-z_{1}\right)\left(c_{A 1}-c_{A 2}\right)}
$$

in which it is advantageous to replace the ratio $E_{t A m} /\left(z_{2}-z_{1}\right)$ with the symbol $F$ (i.e., $F=E_{t A m} /\left(z_{2}-z_{1}\right)$ ) [1]. This is because the quantities $\bar{D}_{T A m}$ and $\left(z_{2}-z_{1}\right)$ are not known at the turbulent fluid flow. These unknowns are replaced by a new quantity, which is determined on the basis of the experimental measurements. According to Eq. (13), the following equation can be written:

$$
F=k_{c} \varphi=\frac{E_{t A m}}{z_{2}-z_{1}}=\frac{D_{A m}+\bar{D}_{T A m}}{z_{2}-z_{1}}
$$


If the bidirectional mass transfer $(\varphi=1)$ occurs in the equipment, based on Eq. (11), the equation is obtained for this case in the form: $N_{A z}=\frac{-E_{t A m}\left(c_{A 2}-c_{A 1}\right)}{z_{2}-z_{1}}$. By comparing this equation with Eq. (12), the following relation can be obtained:

$$
k_{c}^{0}=\frac{E_{t A m}}{z_{2}-z_{1}}=\frac{D_{A m}+\bar{D}_{T A m}}{z_{2}-z_{1}} .
$$

The right sides of Eqs. (14) and (15) are identical, which in the case of the bidirectional mass transfer means that: $k_{c}^{0}=F$.

If $c=$ constant, based on Eq. (10), the following equation can be written: $N_{A z}-\frac{c_{A 1}}{c} \sum_{i=A}^{n} N_{i z}=\left(\frac{c_{A}}{c}-\frac{c_{A 1}}{c}\right) \sum_{i=A}^{n} N_{i z}-E_{t A m} \frac{\mathrm{d} c_{A}}{\mathrm{~d} z}$, whose right side contains the expressions proportional to the difference of the concentrations and concentration gradient, respectively. The concentrations difference, as well as the concentration gradient, represent the driving force of the concentration diffusion process. Therefore, the right side of this equation represents the diffusion flux of substance $A$, which will be further denoted by the symbol $J_{A z}^{\Delta}[6]$. The value of this quantity can again be expressed by an equation similar to Eq. (12), i.e., by the equation:

$$
N_{A z}-\frac{c_{A 1}}{c} \sum_{i=A}^{n} N_{i z}=\left(\frac{c_{A}}{c}-\frac{c_{A 1}}{c}\right) \sum_{i=A}^{n} N_{i z}-E_{t A m} \frac{\mathrm{d} c_{A}}{\mathrm{~d} z}=J_{A z}^{\Delta}=k_{c}^{\Delta}\left(c_{A 1}-c_{A 2}\right),
$$

which is the defining equation for the determination of $k_{c}^{\Delta}$.

The combination of Eqs. (12) and (16) gives the equation:

$$
\frac{k_{c}^{\Delta}}{k_{c}}=\frac{J_{A z}^{\Delta}}{N_{A z}}=1-\frac{c_{A 1}}{c \gamma}=1-\frac{x_{A 1}}{\gamma}
$$

relating the quantities $k_{c}$ and $k_{c}^{\Delta}[6,9]$. The quantity $k_{c}$ expresses the total flux of the substance $i$, the diffuse flux of the substance $i$ (generally represented by molecular and eddy diffusivity) expresses the quantity $k_{c}^{\Delta}$. The convective flux of the substance $i$ is defined by the difference between these two fluxes. In the case of Eq. (16), the value of this flux is given by the expression: $\frac{c_{A 1}}{c} \sum_{i=A}^{n} N_{i z}$.

\section{Relationship among the mass transfer coefficients}

In general, the above-mentioned mass transfer coefficients may have different values and, in a specific case, may have the same value. This is because there is a relation among these mass transfer coefficients, as mentioned above. According to Eq. (16), the following equation, determining the diffuse flux of the substance $i$, may be written: $J_{A z}^{\Delta}=\left(\frac{c_{A}}{c}-\frac{c_{A 1}}{c}\right) \sum_{i=A}^{n} N_{i z}-E_{t A m} \frac{\mathrm{d} c_{A}}{\mathrm{~d} z}$. Using Eq. (17), it is possible to rewrite this equation into the form: 


$$
J_{A z}^{\Delta}=\frac{-E_{t A m}}{\left(\frac{c \gamma-c_{A}}{c \gamma-c_{A 1}}\right)} \frac{\mathrm{d} c_{A}}{\mathrm{~d} z}
$$

whose solution is the equation:

$$
J_{A z}^{\Delta}=\frac{E_{t A m}\left(c \gamma-c_{A 1}\right)}{\left(z_{2}-z_{1}\right) \frac{\left(c_{A 1}-c_{A 2}\right)}{\ln \left(\frac{c \gamma-c_{A 2}}{c \gamma-c_{A 1}}\right)}}\left(c_{A 1}-c_{A 2}\right)=k_{c}^{\Delta}\left(c_{A 1}-c_{A 2}\right) .
$$

This equation is valid in terms of the assumptions already given (i.e., $c=$ constant, $E_{t A m}=$ constant, mass transfer in the direction of the $z$-axis), in which $\gamma=N_{A z} / \sum_{i=A}^{n} N_{i z}$. According to this equation it is possible to write:

$$
k_{c}^{\Delta}=\frac{E_{t A m}\left(c \gamma-c_{A 1}\right) \ln \left(\frac{c \gamma-c_{A 2}}{c \gamma-c_{A 1}}\right)}{\left(z_{2}-z_{1}\right)\left(c_{A 1}-c_{A 2}\right)}=\frac{E_{t A m} \ln \left(1+\frac{c_{A 1}-c_{A 2}}{c \gamma-c_{A 1}}\right)}{\left(z_{2}-z_{1}\right) \frac{\left(c_{A 1}-c_{A 2}\right)}{\left(c \gamma-c_{A 1}\right)}} .
$$

The Eq. (20) can be converted into the form:

$$
\frac{k_{c}^{\Delta}}{k_{c}^{0}}=\frac{k_{c}^{\Delta}}{F}=\frac{\ln (1+R)}{R}=\frac{\Phi}{R}=\theta,
$$

where $\Phi=\ln (1+R)$ (or $\left.R=\mathrm{e}^{\Phi}-1\right)$ and $R=\frac{c_{A 1}-c_{A 2}}{c \gamma-c_{A 1}}=\left(\frac{c_{A 1}}{c \gamma}-\frac{c_{A 2}}{c \gamma}\right) /\left(1-\frac{c_{A 1}}{c \gamma}\right)[6]$.

If Eq. (14) and Eq. (17) are applied in this equation, the following equation is obtained:

$$
\frac{k_{c}\left(1-\frac{c_{A 1}}{c \gamma}\right)}{k_{c}^{0}}=\frac{k_{c}\left(1-\frac{c_{A 1}}{c \gamma}\right)}{F}=\frac{1-\frac{c_{A 1}}{c \gamma}}{\varphi}=\frac{\ln (1+R)}{R}=\frac{\Phi}{R}=\frac{\Phi}{\mathrm{e}^{\Phi}-1}=\frac{k_{c}^{\Delta}}{k_{c}^{0}}=\frac{k_{c}^{\Delta}}{F}=\theta .
$$

This equation expresses the relation among the individual mass transfer coefficients, where $k_{c}^{0}=F$. The dependence of $\theta$ on $\Phi$ is shown in Fig. 2 .

If the bidirectional mass transfer occurs in the equipment, according to Eq. (22), the following applies: $k_{c}=k_{c}^{0}=k_{c}^{\Delta}$, because $1 / \gamma=0, \varphi=1$, and $R=0$. In some special cases, when $c_{A 1} \rightarrow 0$ (or $c_{A 1}-c_{A 2} \rightarrow 0$ ) or $\sum_{i=A}^{n} N_{i z} \rightarrow 0$ applies approximately: $k_{c} \cong k_{c}^{0} \cong k_{c}^{\Delta}$.

It should be noted here that a subscript is usually added to the symbols $R$ and $\Phi[6]$. For example, if the mass transfer takes place in a binary system, $R_{A B}$ and $\Phi_{A B}$ are written instead of $R$ and $\Phi$. 


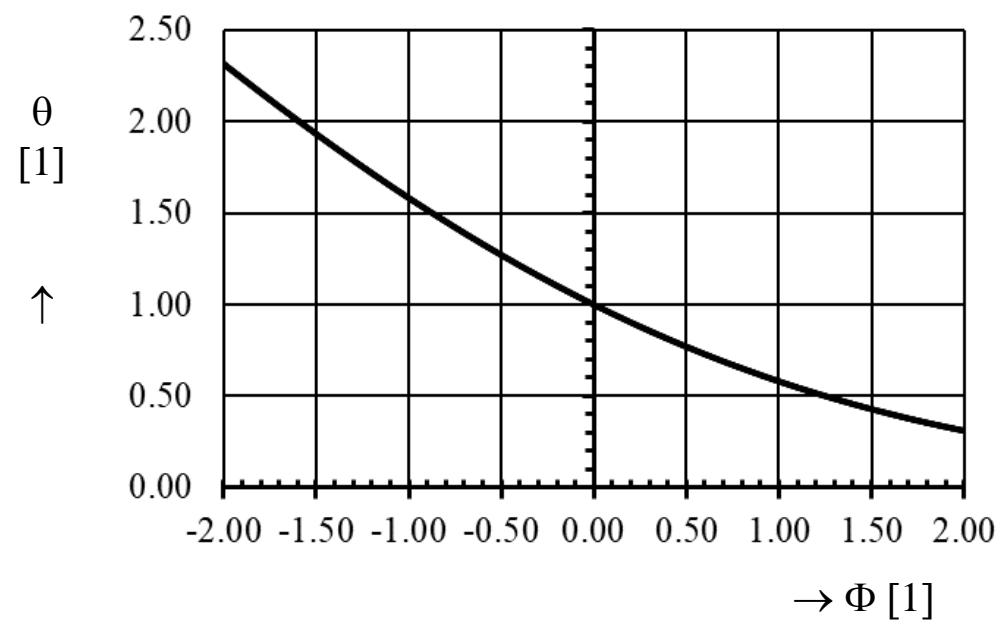

Fig. 2 The dependence of $\theta$ on $\Phi$. The left side of the graph represents the mass transfer of the substance $i$ from the given phase (the substance $i$ leaves the phase, $\Phi$ is a negative value), the right side of the graph represents the mass transfer of the substance $i$ to the given phase (the substance $i$ enters to the phase, $\Phi$ is a positive value).

\section{Mass transfer near the interfacial area}

From the point of view of the quantities labeling, the above-mentioned equations mainly represent the situation that corresponds to the mass transfer in the phase. There are applications in which the substance is transferred from one phase to another. These phases are separated from each other by the interfacial area. For this reason, it is necessary to be able to express the mass transfer of the substance from, or to, the interfacial area. In these cases, the above-mentioned equations, in which different designation of some quantities is usually used than has been used so far, also find the application. The description of the mass transfer of the substance through the interfacial area follows from Fig. (3). This figure expresses the principle of the film model (i.e., film theory of the mass transfer) [5, 9]. In the film model, it is assumed that the concentration change occurs only in the concentration boundary layer, not in the phase volume. The concentration boundary layer, whose thickness is represented by the quantity $\delta_{D}=z_{2}-z_{1}$, is located above the interfacial area. The thickness of this layer depends on the intensity of the fluid turbulence in a given phase. Behind this layer, the concentration in the volume of the phase (i.e., in the volume of the fluid) is considered to be constant (i.e., $c_{A 2}=c_{A f}=$ constant). In the case of mass transfer near the interfacial interface, the initial equation for determining the total molar flux of the substance $A$ is the equation in the form:

$$
\left.N_{A z}\right|_{z=w}=-\frac{E_{t A m} c \gamma}{\left(c \gamma-c_{A}\right)} \frac{\mathrm{d} c_{A}}{\mathrm{~d} z}=N_{A w},
$$

which follows from Eq. (10) provided that $c=$ constant and $E_{t A m}=$ constant. The solution of this equation in the sense of Fig. (3) is the function:

$$
\left.N_{A z}\right|_{z=w}=\frac{-E_{t A m}}{\left(z_{2}-z_{1}\right) \varphi}\left(c_{A f}-c_{A w}\right)=k_{c}\left(c_{A w}-c_{A f}\right)=N_{A w}
$$


in which $k_{c}=\frac{E_{t A m}}{\varphi\left(z_{2}-z_{1}\right)}=\frac{E_{t A m}}{\varphi \delta_{D}}, \varphi=\left(\frac{c_{A w}}{c \gamma}-\frac{c_{A f}}{c \gamma}\right) / \ln \left(\frac{1-\frac{c_{A f}}{c \gamma}}{1-\frac{c_{A w}}{c \gamma}}\right)$, and $1 / \gamma=\sum_{i=A}^{n} N_{i w} / N_{A w}$. Working with the equation of this function is the same as above. Again, it follows from the above that this equation is formally similar to the above-mentioned equations, namely Eq. (11).

The concentration boundary layer represents the total resistance to the mass transfer. In the case of the film model in general, it is also true that $D_{T A m}=\mathrm{f}(z)$. The value of this quantity increases with the distance from the interfacial area at which it is equal to zero (i.e., $D_{T A m}=\mathrm{f}\left(z_{1}\right)=0$ ). Similar conditions are also on the other side of the interfacial area. In other words, the concentration boundary layer exists on both sides of the interfacial area [10]. For this reason, the term two-film theory of mass transfer can also be found in the literature $[11,12,13]$. In these layers, a laminar flow of the substance occurs along the interfacial area. Thus, the laminar film exists in the given phase along the interfacial area. A certain amount of the substance passes through this film at the steady flow of the substance. This amount corresponds to the amount of the substance which is brought mainly as a consequence of the eddy diffusivity (i.e., eddy motion of the fluid) from the volume of the phase to the laminar film. The same amount of the substance must then pass through the interfacial area as well as through the laminar film occurring in the second phase. By transition through this laminar film, the substance subsequently enters the volume of the second phase. In this volume, it is again assumed that the concentration of the substance is constant throughout the volume. This is the essence of the film theory of mass transfer.

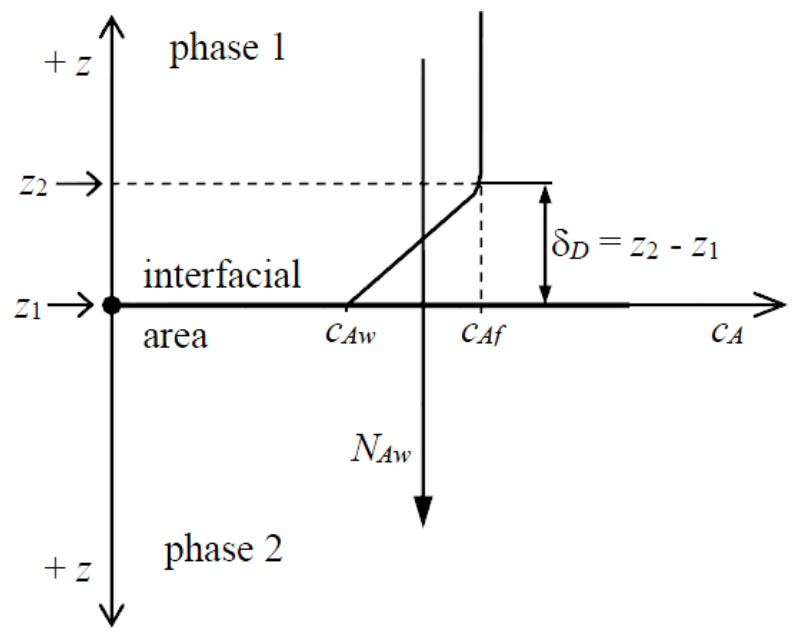

Fig. 3 Illustration of the conditions near the interfacial area in the case of unidirectional mass transfer of substance A.

\section{CONCLUSION}

There are various mass transfer coefficients and therefore it is important to know how these are defined. These coefficients are not possible to confuse with each other. In some cases, it can lead to serious errors in the calculation of the equipment or in comparing the results of the different authors. The article clearly points to the connection among the coefficients $k_{c}^{0}$, $k_{c}^{\Delta}$, and $k_{c}$. Their values are determined mostly experimentally and calculated on the basis of the criterial equations. In most cases, only the coefficient $k_{c}^{0}=F$ is usually determined 
according to the criterial equations. This one must then be recalculated in the sense of the equations mentioned here (for example, according to Eq. (22)) to the assignment conditions. The recalculation is made in order to obtain mainly the value of the coefficient $k_{c}$, alternatively $k_{c}^{\Delta}$. The recalculation need not be done if $\theta=1$ (or $\theta \cong 1$ ). It should also be borne in mind that the individual mass transfer coefficients characterise different substance fluxes. If the mass transfer of the substance $A$ is one-dimensional (for example, along the $z$-axis), the flux of the substance $A$ can be determined according to Eq. (10), alternatively Eq. (11), or from the equation $N_{A z}=J_{A z}^{\Delta}+\frac{c_{A 1}}{c} \sum_{i=A}^{n} N_{i z}$ resulting from Eq. (16). The second equation has the advantage that the value of $J_{A z}^{\Delta}$ can be determined separately in terms of Eq. (19). Subsequently, the value thus determined is added to the value defined in the sense of expression $\frac{c_{A 1}}{c} \sum_{i=A}^{n} N_{i z}$. In both ways, the same value of $N_{A z}$ is obtained. It is worth recalling that these equations are applicable not only in the case of the convective mass transfer, but also in the case of the molecular mass transfer (for which $E_{t A m}=D_{A m}$ ), as well as in the case of mass transfer near the interfacial area.

The equations mentioned here are the basis for the description of the diffusion processes taking place in the equipments and are also used to describe the mass transfer of a substance in the solid material. In such cases, the diffusivity coefficient is replaced by the effective diffusivity coefficient $\left(D_{e f}\right)$. The above mentioned equations also apply in the case of systems with chemical reactions. It follows that it is important to understand the equations presented here, because their knowledge is a necessary condition for understanding other equations, for the creation of which the equations mentioned here also apply. In conclusion, it should be noted, the equations defining the mass transfer coefficients here make it possible to determine the local values of these coefficients. If the mass transfer coefficient in the equipment changes with position, the mean value of this coefficient should be considered in the calculation.

\section{ACKNOWLEDGEMENTS}

This research did not receive any specific grant from funding agencies in the public, commercial, or not-for-profit sectors.

\section{Nomenclature}

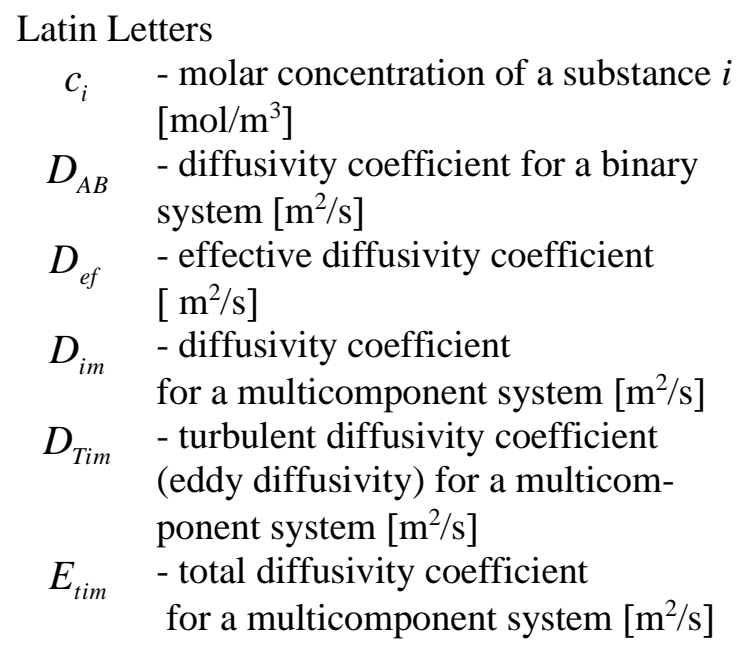

$J_{i} \quad$ - molar flux of a substance $i$ (total molar flux of a substance $i$ ), including the convective flux of substance $i$ $\left[\mathrm{mol} /\left(\mathrm{m}^{2} . \mathrm{s}\right)\right]$

$J_{i^{\prime}} \quad$ - molar flux of a substance $i$ due to the existence of a molecular flux of a substance $i\left[\mathrm{~mol} /\left(\mathrm{m}^{2} . \mathrm{s}\right)\right]$

$J_{i "} \quad$ - molar flux of a substance $i$ due to the existence of a convective flux of a substance $i\left[\mathrm{~mol} /\left(\mathrm{m}^{2} . \mathrm{s}\right)\right]$

$k_{c} \quad$ - mass transfer coefficient based on a molar concentration differences $[\mathrm{m} / \mathrm{s}]$ 


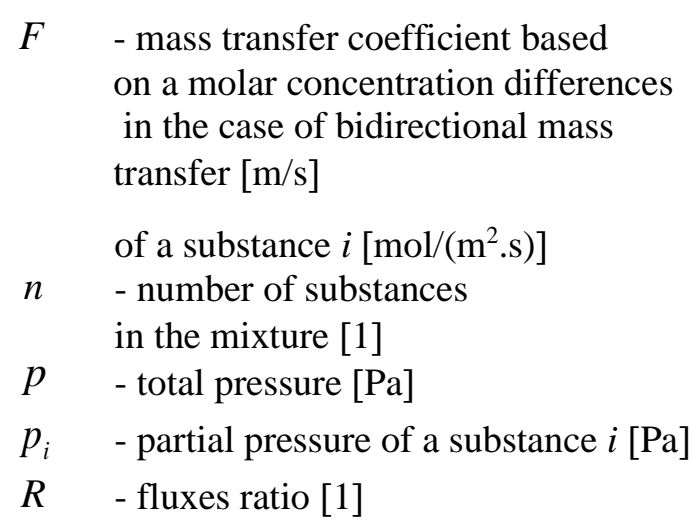

Greek letters

$$
\begin{array}{cl}
\beta_{A B} & \text { - Maxwell diffusivity coefficient } \\
& \text { for binary system [kg.m } \left.3 /\left(\mathrm{s} \cdot \mathrm{mol}^{2}\right)\right] \\
\delta_{D} & \text { - boundary layer thickness [m] } \\
\Phi & \text { - rate factor [1] }
\end{array}
$$

Mathematical operations

$$
\nabla \quad \text { - del operator }[1 / \mathrm{m}]
$$

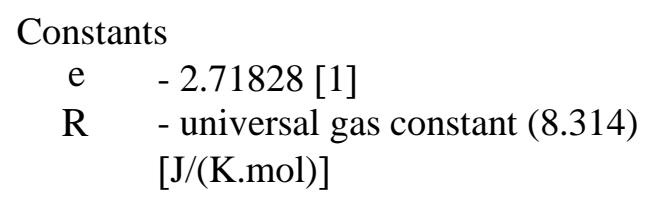

Overlines

$$
\vec{X} \quad \text { - vector }
$$

\section{Superscripts}

$$
\begin{array}{cl}
X^{*} & \text { - molar } \\
X^{\prime} & \begin{array}{l}
\text { - used for variables corresponding } \\
\text { to molecular mass transfer }
\end{array} \\
X^{0} & \begin{array}{l}
\text { - used for variables corresponding } \\
\text { to bidirectional mass transfer }
\end{array} \\
\left.X\right|_{z=w} & \text { - near the interfacial area }
\end{array}
$$

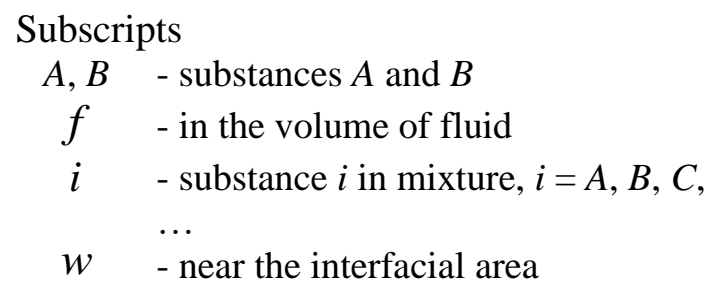

$N_{i} \quad$ - total molar flux of a substance $i$ $\left[\mathrm{mol} /\left(\mathrm{m}^{2} . \mathrm{s}\right)\right]$

$N_{i^{\prime \prime}} \quad$ - molar flux of a substance $i$ due to the existence of a convective flux

$u$ - average velocity of the mixture $[\mathrm{m} / \mathrm{s}]$

$u_{i} \quad$ - local velocity of a substance $i[\mathrm{~m} / \mathrm{s}]$

$x_{i} \quad$ - molar fraction of a substance $i$ [1]

$z \quad$ - distance from the interfacial area [m]
$\gamma \quad$ - the ratio of molar fluxes [1]
$\varphi \quad$ - factor taking into account the type of concentration diffusion [1]
$\theta \quad$ - correction factor [1]


[4] Wankat, P. C. "Separation Process Engineering Includes Mass Transfer Analysis", Prentice Hall, 2012, ISBN-978-0-13-138227-5, p. 958.

[5] Madlo, V. "Teoretické základy odboru”, Vydavatel'stvo STU v Bratislave, 1994, ISBN-80-2270663-9, p. 313.

[6] Bird, R. B., Stewart, W. E., Lightfoot, E. N. "Transport Phenomena", John Wiley \& Sons, Inc., 2002, ISBN-0-471-41077-2, p. 895.

[7] Karas, I., Pajslhauser, L. "Difúzne pochody pre zameranie chemické stroje", Edičné stredisko SVŠT v Bratislave, Edičné číslo 2893, 1984, p. 201.

[8] Gužela, Š. “Látkové bilancie chemických procesov", Vydavatel'stvo STU, 2008, ISBN-978-80227-2961-1, p. 224.

[9] Míka, V. “Základy chemického inženýrství”, SNTL/ALFA, 1981, Číslo 04-605-81, p. 872.

[10] Kossaczký, E., Surový, J. “Chemické inžinierstvo 2”, ALFA, 1983, Č́slo 63-564-83, p. 400.

[11] Wang, C., Xu, Z., Lai, C., Sun, X. "Beyond the Standard Two-Film Theory: Computational Fluid Dynamics Simulations for Carbon Dioxide Capture in a Wetted Wall Column", Chemical Engineering Science 184, pp. 103 - 110, 2018. DOI: 10.1016/j.ces.2018.03.021.

[12] Almoslh, A., Alobaid, F., Heinze, Ch., Epple, B. "Influence of Pressure on Gas/Liquid Interfacial Area in a Tray Column", Applied Sciences 10 (4617), p. 17, 2020. DOI:10.3390/app10134617.

[13] Chikukwa, A., Enaasen, N., Kvamsdal, H. M., Hillestad, M. "Dynamic Modeling of Postcombustion CO2 Capture Using Amines - A Review", Energy Procedia 23, pp. 82 - 91, 2012. DOI: $10.1016 /$ j.egypro.2012.06.063. 